PAPER

\title{
Neuropsychiatric profiles in patients with Alzheimer's disease and vascular dementia
}

\author{
J-L Fuh, S-J Wang, J L Cummings
}

J Neurol Neurosurg Psychiatry 2005;76:1337-1341. doi: 10.1136/jnnp.2004.056408

See end of article for authors' affiliations

.....................

Correspondence to:

Dr J-L Fuh, Neurological

Institute, Taipei Veterans

General Hospital, Taiwan,

Republic of China; j|fuh@

vghtpe.gov.tw

Received 14 October 2004

In revised form

16 January 2005

Accepted 26 January 2005
Objective: To explore the neuropsychiatric manifestations in patients with Alzheimer's disease (AD) and cortical and subcortical vascular dementia (VaD).

Methods: We investigated consecutive patients with dementia. All the participants received brain computed tomography. The diagnosis of dementia was confirmed by clinical criteria and the imaging findings. Only patients with probable AD, and subcortical and cortical VaD were included. The Mini Mental State Examination (MMSE) was used to evaluate global cognitive function, and the Neuropsychiatric Inventory (NPI) was used to assess neuropsychiatric symptoms.

Results: Of the 536 participants with dementia, 320 (59.7\%) had AD, 161 (30\%) had subcortical VaD, 35 (6.4\%) had cortical VaD, and 16 (2.9\%) had mixed cortical and subcortical VaD. Cortical VaD patients had the highest mean composite NPI scores in all domains and AD patients had the lowest composite scores in most domains. The mean composite scores of the apathy and sleep disturbance domains in patients with cortical $\mathrm{VaD}$ were significantly higher than those in the patients with $A D$ after controlling for years of education and MMSE score $(p<0.01)$.

Conclusions: There were few differences among the patients with $A D$, subcortical VaD and cortical VaD. The most consistent differences were the high sleep disturbance scores in those with cortical VaD.
$\mathrm{N}$ europsychiatric symptoms can induce marked disability in patients with dementia and increase caregiver distress. ${ }^{1}$ Several studies have found that neuropsychiatric symptoms are common both in patients with Alzheimer's disease (AD) and those with vascular dementia (VaD). ${ }^{2-6}$ Nevertheless, studies of the neuropsychiatric disturbances in patients with VaD are few and the results are controversial. Some studies suggested that neuropsychiatric symptoms were more common in VaD than in $\mathrm{AD}$, but others report conflicting observations. ${ }^{2-4}$ Investigations of affective disorder also revealed controversial findings between the patients with $\mathrm{VaD}$ and $\mathrm{AD} .^{25}$ These discrepancies might be caused by different VaD diagnostic criteria with variable sensitivity and specificity, ${ }^{7-10}$ and use of different evaluation tools for neuropsychiatric manifestations.

The association between $\mathrm{AD}$ and $\mathrm{VaD}$ is complex. It is not uncommon to have vascular changes in patients with $\mathrm{AD}$, and the vascular lesions can worsen cognitive impairment. ${ }^{11-13}$ Nevertheless, the clinical cognitive profiles of $\mathrm{AD}$ and $\mathrm{VaD}$ are distinguishable, as frontal executive dysfunction is the most prominent feature of VaD. ${ }^{14}$ Could different types of dementia also present different neuropsychiatric profiles? This question is important because these differences could affect treatment approaches and caregiver distress. ${ }^{6}{ }^{15}$

The neuropathology of $\mathrm{VaD}$ is heterogeneous. Several vascular pathologies can lead to dementia. ${ }^{16}$ The clinical presentation and course of these different subtypes of VaD vary but their neuropsychiatric manifestations have rarely been studied. A syndrome of subcortical VaD incorporating both "Binswanger's disease" and "lacunar state" is a more homogenous group. ${ }^{17}$ To subgroup $\mathrm{VaD}$ into subcortical and cortical types may facilitate comparisons between $\mathrm{AD}$ and VaD.

In this study, we assessed neuropsychiatric symptoms using a standard tool, the Neuropsychiatric Inventory (NPI), ${ }^{18}$ in a large clinical sample of consecutive patients with $\mathrm{AD}$ and different subtypes of VaD. The aim of this study was to determine if the neuropsychiatric manifestations in patients with $\mathrm{AD}$, cortical VaD, and subcortical VaD differ.

\section{PATIENTS AND METHODS \\ Patients}

The study comprised 1376 consecutive outpatients who complained of memory decline and were examined at the outpatient clinic of the Taipei Veterans General Hospital from December 1999 to October 2003. The Institutional review board of Taipei Veterans General Hospital approved the study protocol.

\section{Assessments and measures}

All patients were evaluated by clinical interview, and neurological and physical examination. The Mini Mental State Examination (MMSE) ${ }^{19}$ and $\mathrm{NPI}^{18}$ were administered to the patients and caregivers respectively.

The $\mathrm{MMSE}^{19}$ is one of the most widely used screening instruments for dementia, and provides a total score ranging from 0 to 30, with lower scores indicative of greater cognitive impairment. It was administered to the patients to obtain an overall level of current cognitive function.

Information on neuropsychiatric symptoms was obtained from caregivers using the NPI. ${ }^{18}$ There is a screening question assaying each sub-area, including delusions, hallucinations, agitation, apathy, anxiety, depression, euphoria, irritability, disinhibition, aberrant motor behaviour, change in appetite, and night time behaviour disturbances. If the answer to this screening question was "no", then no further questions were asked. If the answer was "yes", then sub-questions were asked and ratings of the frequency and severity of the

Abbreviations: $A D$, Alzheimer's disease; CDR, Clinical Dementia Rating; CT, computed tomography; CVD, cerebrovascular diseases; DSM-IV, Diagnostic and statistical manual of mental disorders, 4th edition; MMSE, Mini Mental State Examination; NINCDS-ADRDA, National Institute of Neurological and Communicative Disorders and Stroke-Alzheimer's Disease and Related Disorders Association; NPI, Neuropsychiatric Inventory; $\mathrm{VaD}$, vascular dementia 
behaviour were made by the caregiver based on scales with anchor points (frequency: $1=$ occasionally, $2=$ often, $3=$ frequently, 4 = very frequently; severity: $1=$ mild, 2 = moderate, $3=$ severe). The frequency rating multiplied by the severity rating produced a composite score for each behaviour. A global score for the NPI was generated by summing the total scores of the individual subscales. Cronbach's $\alpha$ for overall reliability of NPI was 0.88 and the concurrent validity was good, as shown by an acceptable correlation between NPI scores and other validated measurements. ${ }^{18}$

The Clinical Dementia Rating (CDR) scale provides a global rating of the severity of dementia, ranging from 0 (no impairment) to 3 (severe impairment). ${ }^{20}$ The overall CDR rating was obtained from one primary domain (memory) and five secondary domains (orientation, judgement and problem solving, community affairs, home hobbies, and personal care).

We reviewed the relevant medical records and requested laboratory tests for participants with dementia, including complete blood counts, chemistries, serum vitamin B12, folic acid, thyroid function tests, and syphilis serology. Brain computerised tomography (CT) was also obtained on all participants with dementia.

\section{Criteria of dementia}

The diagnosis of dementia was made according to the Diagnostic and statistical manual of mental disorders, 4th edition (DSM-IV) criteria. ${ }^{21}$ Patients with delirium were excluded.

Subjects who met the dementia criteria were further classified as to their type of dementia at a consensus meeting after the history, laboratory findings, and the results of brain CT were reviewed. The diagnostic physicians were blinded to the results of the NPI.

Patients were diagnosed as having $\mathrm{AD}$ if they met the criteria of the National Institute of Neurological and Communicative Disorders and Stroke-Alzheimer's Disease and Related Disorders Association (NINCDS-ADRDA) guidelines. $^{22}$ Patients who fulfilled NINCDS-ADRDA ${ }^{23}$ criteria for probable $\mathrm{AD}$ and had a single "silent" lacune, small cortical infarct, or disproportionately large ventricles found on brain imaging were excluded from analysis in this study.

VaD was defined according to modified DSM-IV criteria. ${ }^{21}$ DSM-IV criteria ${ }^{21}$ for VaD originally allowed focal neurological signs or laboratory evidence indicative of cerebrovascular disease (CVD). However, in this study, we required both the clinical criteria and demonstrate ischaemic abnormalities on CT. We classified VaD based on CT findings as subcortical VaD (small vessel disease, multiple lacunar infarcts, and/or extensive periventricular white matter lesions) and cortical VaD (large vessel disease, infarcts involving the cortical grey matter). We excluded patients with cerebral haemorrhage.

\section{Statistical analysis}

Statistical analysis was performed with SPSS for Windows (version 11.0.; SPSS Inc., Chicago, IL, USA) We used the $\chi^{2}$ test to compare the proportions of participants with different types of dementia who had a score of 1 or higher in any individual NPI domain. We also compared the prevalence of neuropsychiatric disturbances in different dementia stages. We compared the NPI mean scores for participants with AD, subcortical VaD, cortical VaD, and mixed cortical and subcortical $\mathrm{VaD}$ as an indicator of behavioural change in each domain. The differences between the groups were analysed using one way analysis of variance, and the Scheffé test was used to explore the individual group effects.

To control for the severity of dementia as a confounding factor, we constructed a series of linear regression models, one for each domain and one for the total NPI score. Individual NPI domain scores or the NPI total score were the dependent variables, and the presence or absence of different types of dementia was the independent variable. We also adjusted for MMSE and educational level in the analysis. A p value $<0.01$ was regarded as statically significant, balancing the risk of type I and II errors.

\section{RESULTS}

\section{Patients' characteristics}

Fig l shows a flow diagram describing the number of participants participating to the study and those meeting inclusion and exclusion criteria. A total 536 subjects participated in this study: 320 (59.7\%) with $\mathrm{AD}$ (mean (SD) age $75.2(7.2)$ years), 161 (30\%) with subcortical VaD (75.1 (7.3) years), 35 (6.4\%) with cortical VaD (75.8 (6.9) years), and $16(2.9 \%)$ with mixed cortical and subcortical VaD (74.0 (7.0) years). The proportion of male patients was significantly lower in the AD compared with the subcortical VaD group $(54 \% \vee 69 \%, \mathrm{p}<0.01)$ and the proportion of severe dementia $(\mathrm{CDR}=3)$ was significantly lower in the $\mathrm{AD}$ compared with the cortical VaD group $(8 \% v$ $29 \%, \mathrm{p}<0.001$ ). The age, educational level, and MMSE score did not differ between the subjects with solely cortical and with mixed cortical and subcortical lesions. The patients with solely cortical VaD had significantly lower mean (SD) MMSE scores compared with $\mathrm{AD}$ or solely subcortical VaD patients (11.6 (7.5) $v 17.1$ (6.8) and $15.6(6.8), \mathrm{p}<0.001)$.

\section{Neuropsychiatric manifestations among the different types of dementia}

Fig 2 compares the prevalence of NPI based disturbances in participants with $\mathrm{AD}$ and those with subcortical, cortical, and mixed VaD. The prevalence of NPI disturbances was similar in the four groups. The patients with cortical VaD had marginally greater prevalence of agitation and sleep disturbances $(p=0.03$ and 0.04 , respectively).

Comparing the mean composite subcores of the NPI domains across the four types of dementia, the cortical VaD had the highest composite scores in all domains and the highest total NPI score. The total 12 item NPI score reached significance by one way analysis of variance $(\mathrm{p}<0.01)$ but showed only a trend toward a difference between $\mathrm{AD}$ and cortical VaD by the Scheffé test (mean (SD) 13.8 (17.9) v 24.6 (27.3), $\mathrm{p}=0.02$ ). Among the subdomains, only the apathy and sleep disturbance domains reached statistical significance by one way analysis of variance $(\mathrm{p}<0.01)$. Comparisons using the Scheffé test indicated that the cortical VaD group had significantly higher scores compared with the AD group in the sleep disturbance domain (3.6 (4.1) $v$ l.7 (2.8), $\mathrm{p}<0.01$ ), and there was a trend for patients with cortical VaD and subcortical VaD to have higher scores compared with the AD group in the apathy domain (3.4 (3.9) and 2.6 (3.8) $v 1.6$ (2.8), $\mathrm{p}=0.03)$.

\section{Relationship of severity of dementia to the neuropsychiatric manifestations}

Table 1 shows the prevalence of individual NPI domain scores in patients with dementia of different severities as classified by the CDR. We did not include mixed VaD during the analysis because of the small number of patients. The prevalence of delusions, hallucinations, and aberrant motor activities increased significantly with the severity of dementia in patients with $\mathrm{AD}$ and subcortical VaD. The differences were statistically significant for the prevalence of apathy, agitation, and disinhibition in patients with $\mathrm{AD}$. The prevalence of sleep disturbance was different in the patients with subcortical VaD. There were no differences in the prevalence of any NPI domain except the apathy domain across CDR staging in patients with cortical VaD. In patients with mild dementia $(C D R=1)$, there were no differences in 


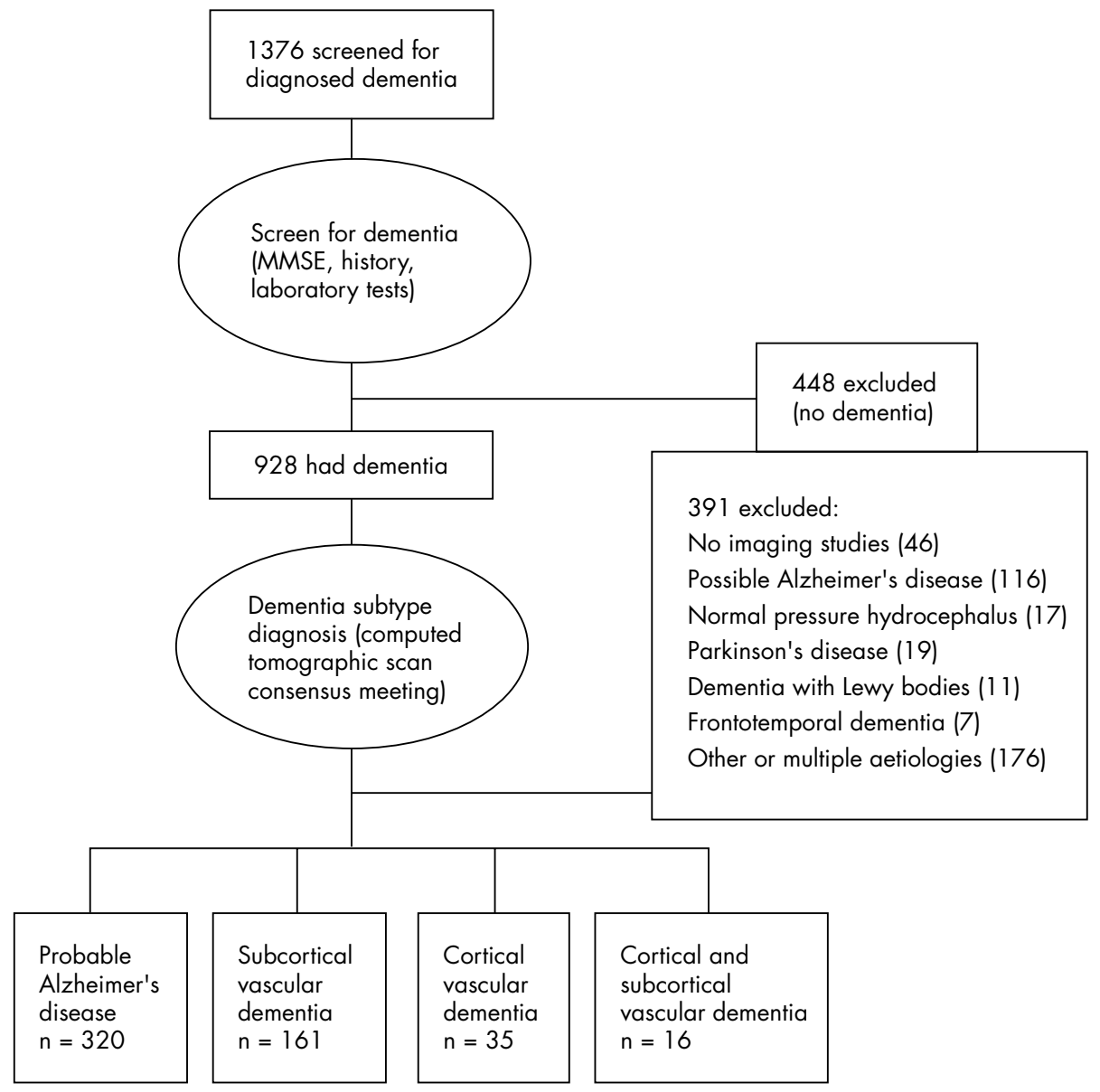

Figure 1 Flow diagram of participants meeting inclusion and exclusion criteria.

the prevalence of any NPI domain across different types of dementia. Although the prevalence of anxiety differed among different dementia types, the domain scores were not significantly different, owing to the small number of cortical VaD group.

As the prevalence of NPI disturbances might be confounded by the severity of dementia, and the cortical VaD patients had the lowest MMSE score and educational level, we conducted a linear regression analysis to compare the apathy score in each of the three groups after controlling for these two factors. The patients with cortical VaD still had higher mean scores than the patients with $\mathrm{AD}$ in the apathy $(\beta=0.12, \quad t=2.75, \quad p=0.006)$ and sleep disturbance $(\beta=0.13, t=2.89, p=0.004)$ domains. The total 12 item NPI score did not show significant differences among the different types of dementia after controlling for MMSE and educational level $(\beta=0.10, t=2.29, p=0.02)$

\section{DISCUSSION}

The present study demonstrated that behavioural disturbances are very common in patients with dementia, regardless of the dementia type. Consistent with previous reports, ${ }^{23-25}$ our data showed only minor differences in the prevalence of behavioural disturbances in $\mathrm{AD}$ and VaD. It also supported past clinical reports that the differentiation of $\mathrm{VaD}$ from $\mathrm{AD}$ is difficult using clinical and neuropsychiatric features only. ${ }^{5}$ Nevertheless, specific differences in neuropsychiatric manifestations existed between patients with $\mathrm{AD}$ and $\mathrm{VaD}$, most prominently in the domain of sleep disturbance, which was more severe in cortical VaD. Our study also revealed a trend for the severity of neuropsychiatric manifestations to be greatest in cortical VaD and least in AD. Our study results emphasise the importance of subgrouping VaD into cortical and subcortical types.

Patients with cortical VaD had significantly higher mean composite scores in the sleep disturbance domain. This is consistent with a previous small group studies showing that VaD patients have more disrupted sleep/wake cycles and decreased sleep quality compared with $\mathrm{AD}$ patients. ${ }^{26}$

Our study showed that patients with VaD tended to have higher scores on the apathy domain compared with $\mathrm{AD}$ patients, although the differences did not reach statistical significance. Apathy has been attributed to disruption of corticosubcortical circuits involving the basal ganglia, thalamus, and frontal lobes. ${ }^{27-29} \mathrm{AD}$ is a cortical dementia, and the subcortical structures are largely preserved, 3031 whereas subcortical VaD disturbs corticosubcortical circuits with lacunar lesions and white matter ischaemic injury. Cortical lesions in VaD likewise commonly involve frontal regions and may disrupt corticosubcortical circuits mediating motivational behaviour.

Studies investigating the association of neuropsychiatric manifestations with dementia severity have failed to consistently demonstrate differences in $\mathrm{AD}$ compared with VaD. ${ }^{152432} 33$ We found that some disturbances such as delusions, hallucinations, and aberrant motor activities were more common in later stages in both patients with $\mathrm{AD}$ and those with subcortical VaD. A large community based study found agitation and aberrant motor behaviour to be more common in participants with advanced dementia. ${ }^{24}$ Our findings support this observation; we found agitation to be more common in later stages among patients with $\mathrm{AD}$ but 


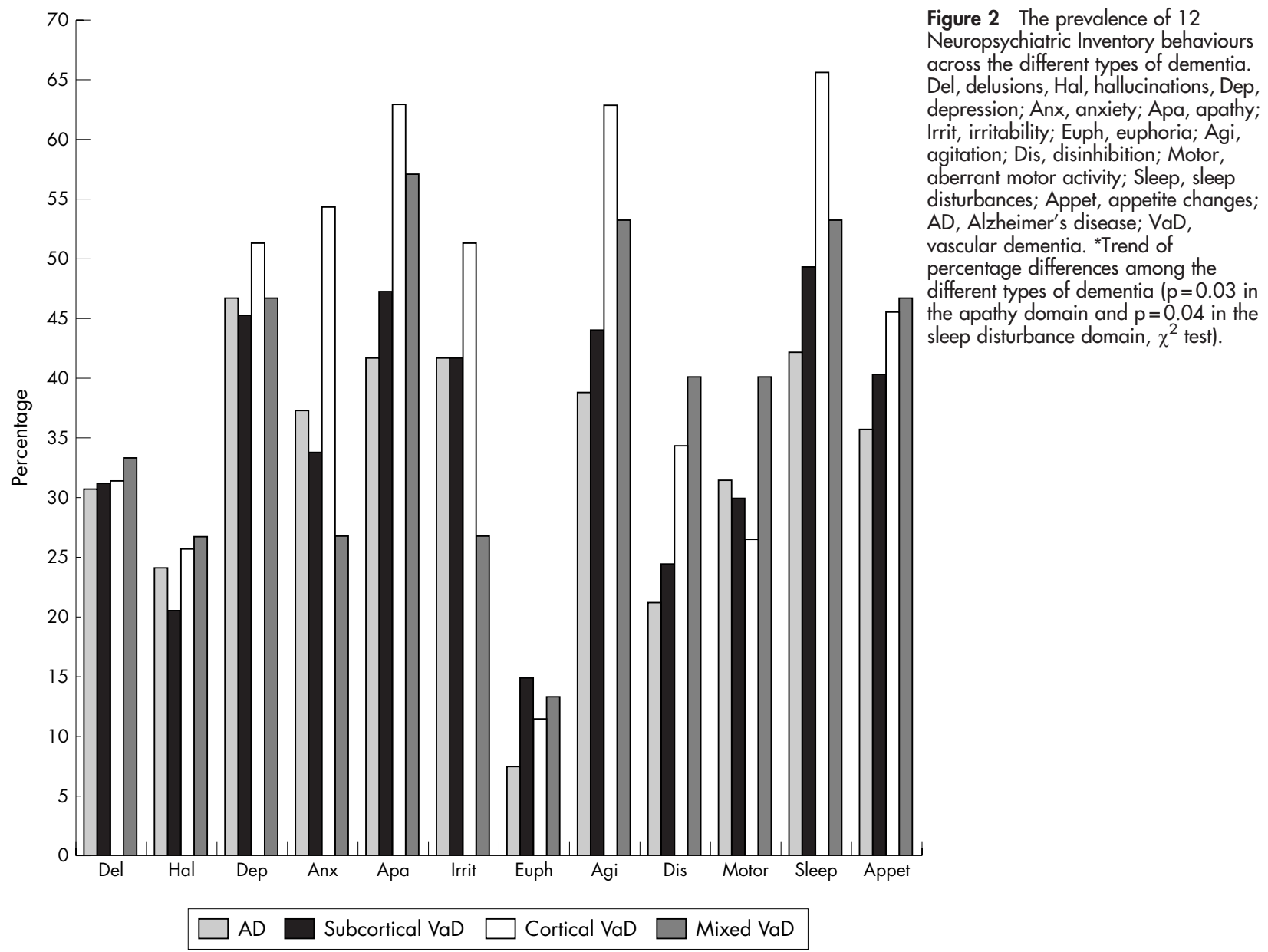

not VaD. The higher prevalence of neuropsychiatric manifestations in our patients may be attributable to differences in study populations (hospital based versus community based).

The strength of this study is that it was a large, prospective study using a standardised assessment tool and concomitant CT. However, there are some limitations. Firstly, because subjects who participated in this study were from a medical centre, caution should be taken in generalising the study results. Secondly, the rate of diagnostic misclassification was uncertain because of the lack of neuropathological confirmation in our study. In this study, we attempted to identify "pure" AD and excluded subjects who fulfilled the NINCDS$\mathrm{ADRDA}^{22}$ but also had vascular lesions on brain CT. For VaD, we used the DSM-IV criteria $^{21}$ in addition to brain CT for diagnosis. Imaging may help compensate for the relatively low specificity of DSM-IV criteria for VaD. ${ }^{21}$ Mixed cases may

Table 1 Percentage frequency of Neuropsychiatric Inventory disturbances in patients with different types dementia classified by dementia severity

\begin{tabular}{|c|c|c|c|c|c|c|c|c|c|c|c|c|}
\hline \multirow[b]{2}{*}{ NPI domain } & \multicolumn{3}{|l|}{$C D R=1$} & \multicolumn{3}{|l|}{$\mathrm{CDR}=2$} & \multicolumn{3}{|l|}{$C D R=3$} & \multicolumn{3}{|l|}{ Total } \\
\hline & $\begin{array}{l}\text { AD } \\
(n=188)\end{array}$ & $\begin{array}{l}\text { sVaD } \\
(n=82)\end{array}$ & $\begin{array}{l}c V a D \\
(n=11)\end{array}$ & $\begin{array}{l}A D \\
(n=107)\end{array}$ & $\begin{array}{l}\text { sVaD } \\
(n=59)\end{array}$ & $\begin{array}{l}\text { cVaD } \\
(n=14)\end{array}$ & $\begin{array}{l}\text { AD } \\
(n=25)\end{array}$ & $\begin{array}{l}\text { sVaD } \\
(n=20)\end{array}$ & $\begin{array}{l}\text { cVaD } \\
(n=10)\end{array}$ & $\begin{array}{l}\text { AD } \\
(n=320)\end{array}$ & $\begin{array}{l}\text { sVaD } \\
(n=161)\end{array}$ & $\begin{array}{l}\text { cVaD } \\
(n=35)\end{array}$ \\
\hline Delusions ${ }^{*} \dagger$ & 19.9 & 14.1 & 9.1 & 43.9 & 45.9 & 35.7 & 58.3 & 60.9 & 50.0 & 31.0 & 31.1 & 31.4 \\
\hline Hallucinations ${ }^{*} \dagger$ & 13.9 & 21.6 & 18.2 & 34.6 & 24.6 & 14.3 & 58.3 & 60.9 & 50.0 & 24.1 & 21.0 & 25.7 \\
\hline Depression & 46.3 & 39.1 & 45.5 & 44.3 & 49.2 & 42.9 & 54.2 & 47.8 & 70.0 & 46.7 & 45.0 & 51.0 \\
\hline Anxiety & 32.6 & 28.3 & 63.5 & 43.0 & 38.3 & 42.9 & 44.0 & 52.2 & 60.0 & 37.2 & 33.8 & 51.0 \\
\hline Apathy* $\ddagger$ & 43.6 & 42.4 & 36.4 & 55.1 & 49.2 & 57.1 & 64.0 & 60.9 & 100 & 41.6 & 47.2 & 62.9 \\
\hline Irritability & 38.0 & 42.4 & 36.4 & 45.8 & 36.1 & 50.0 & 50.0 & 60.9 & 70.0 & 42.0 & 41.6 & 51.4 \\
\hline Euphoria & 5.3 & 12.0 & 9.1 & 9.3 & 13.1 & 7.1 & 16.0 & 30.4 & 20.0 & 7.5 & 14.9 & 11.0 \\
\hline Agitation/aggression* & 28.9 & 39.1 & 45.5 & 49.5 & 47.5 & 71.4 & 64.0 & 52.2 & 70.0 & 38.8 & 44.1 & 62.9 \\
\hline Disinhibition* & 13.4 & 18.5 & 9.1 & 32.1 & 26.2 & 35.7 & 32.0 & 45.5 & 60.0 & 21.1 & 24.0 & 34.3 \\
\hline Aberrant motor & 17.2 & & 18.2 & 49.1 & & 23.1 & & & 40.0 & 31.4 & & \\
\hline activity ${ }^{*} \dagger$ & & 18.5 & & & 35.0 & & 60.0 & 65.2 & & & 30.0 & 26.5 \\
\hline Sleep disturbance $†$ & 36.9 & 38.5 & 63.6 & 47.7 & 57.4 & 64.3 & 56.0 & 69.6 & 70.0 & 42.2 & 49.4 & 65.7 \\
\hline Appetite change & 30.5 & 34.1 & 45.5 & 40.6 & 39.3 & 35.7 & 52.2 & 63.6 & 60.0 & 35.6 & 40.3 & 45.7 \\
\hline
\end{tabular}

NPI, Neuropsychiatric Inventory, CDR, clinical dementia rating, AD, Alzheimer's disease, sVaD, subcortical vascular dementia, cVaD, cortical vascular dementia. *Significant difference in prevalence of different CDR group among patients with Alzheimer disease $\left(p<0.01, \chi^{2}\right.$ test); $†$ significant difference in prevalence of different CDR group among patients with subcortical vascular dementia $\left(p<0.01, \chi^{2}\right.$ test); $\ddagger$ significant difference in prevalence of different CDR group among patients with cortical vascular dementia $\left(\mathrm{p}<0.01, \chi^{2}\right.$ test). 
be present in the sample despite these diagnostic procedures Thirdly, the relatively small sample size of patients with cortical VaD might cause some differences among behavioural domains to fail to reach statistical significance. It is also possible that a few patients with very severe psychopathology had a large influence on the small number of samples and thus biased the study results.

In conclusion, the present study showed a high prevalence of neuropsychiatric symptoms in both $\mathrm{AD}$ and $\mathrm{VaD}$. Symptoms profiles were similar in the AD, subcortical VaD, and cortical VaD groups. Sleep disturbance was most severe in the patients with cortical VaD.

\section{ACKNOWLEDGEMENTS}

This study was supported in part by grants from National Science Council (NSC- 92-2314-B-075-033).

\section{Authors' affiliations}

J-L Fuh, S-J Wang, Neurological Institute, Veterans General Hospital Taipei and National Yang-Ming University Schools of Medicine, Taipei, Taiwan

J L Cummings, UCLA Alzheimer's Disease Center, Departments of Neurology and Psychiatry, David Geffen School of Medicine at UCLA, Los Angeles, California, USA

Competing interests: none declared.

\section{REFERENCES}

1 Rabins PV, Mace NL, Lucas MJ. The impact of dementia on the family. JAMA 1982;248:333-5.

2 Sultzer DL, Levin HS, Mahler HW, et al. A comparison of psychiatric symptoms in vascular dementia and Alzheimer's disease. Am J Psychiatry 1993;150:1806-12.

3 Bucht G, Adolfson R. The comprehensive psychopathological rating scale in patients with dementia of Alzheimer's type and multi-infarct dementia. Acto Psychiatr Scand 1983:68:263-70.

4 Swearer JM, Drachman DA, O'Donnell BF, et al. Troublesome and disruptive behaviors in dementia: relationship to diagnosis and disease severity. J Am Geriatr Soc 1988;36:784-90.

5 Groves WC, Brandt J, Steinberg M, et al. Vascular dementia and Alzheimer's disease: is there a difference? A comparison of symptoms by disease duration. J Neuropsychiatry Clin Neurosci 2000;12:305-15.

6 Vetter PH, Krauss S, Steiner O, et al. Vascular dementia versus dementia of Alzheimer's type: do they have differential effects on caregivers' burden? J Gerontol B Psychol Sci Soc Sci 1999;54:S93-8.

7 Wetterling T, Kanitz RD, Borgis KJ. Comparison of different diagnostic criteria for vascular dementia (ADDTC, DSM-IV, ICD-10, NINDS-AIREN). Stroke 1996;27:30-6.

8 Chui HC, Mack W, Jackson JE. Clinical criteria for the diagnostic of vascular dementia: a multi-centre study of comparability and inter-rater reliability. Arch Neurol 2000;57:1295-300.

9 Pohjasvaara T, Mantyla R, Salonen O. How complex interactions of ischemic brain infarcts, white matter lesions, and atrophy relate to poststroke dementia. Arch Neurol 2000;57:191-6.
10 Gold G, Bouras C, Canuto A. Clinicopathological validation study of four sets of clinical criteria for vascular dementia. Am J Psychiatry 2002;159:82-7.

11 Goulding JM, Signorini DF, Chatterjee S, et al. Inverse relation between Braak stage and cerebrovascular pathology in Alzheimer predominant dementia. J Neurol Neurosurg Psychiatry 1999:67:654-7.

12 Esiri MM, Nagy Z, Smith MZ, Barnetson L, Smith AD. Cerebrovascular disease and threshold for dementia in the early stages of Alzheimer's disease. Lancet 1999;354:919-20

13 Snowdon DA, Greiner LH, Mortimer JA, et al. Brain infarction and the clinical expression of Alzheimer disease. The Nun study. JAMA 1997:277:813-17.

14 Looi JC, Sachdev PS. Differentiation of vascular dementia from AD on neuropsychological tests. Neurology 1999;53:670-8.

15 Kim JM, Lyons D, Shin JS, et al. Differences in behavioral and psychological symptoms between Alzheimer's disease and vascular dementia: Are the different pharmacologic treatment strategies justifiable? Hum Psychopharmacol 2003;18:215-20.

16 Brien JT, Erkinjuntti T, Reisberg B, et al. Vascular cognitive impairment. Lancet Neurol 2003;2:89-98.

17 Erkinjuntti T, Inzitari D, Pantoni L, et al. Limitations of clinical criteria for the diagnosis of vascular dementia in clinical trials: is a focus on subcortical vascular dementia a solution? Ann N Y Acad Sci 2000;903:262-72.

18 Cummings JL, Mega M, Gray K, et al. The Neuropsychiatric Inventory: comprehensive assessment of psychopathology in dementia. Neurology 1994:44:2308-14.

19 Folstein MF, Folstein SE, McHugh PR. Mini-Mental State. A practical method for grading the cognitive state of patients for clinician. J Psychiat Res 1975;12:189-98.

20 Morris JC. The Clinical Dementia Rating (CDR): current version and scoring rules. Neurology 1993;43:2412-14.

21 American Psychiatric Association. Diagnostic and statistical manual of mental disorders, 4th ed. Washington, DC: American Psychiatric Association, 1994.

22 McKhann G, Drachman D, Folstein M et al. Clinical diagnosis of Alzheimer's disease: report of the NINCDS-ADRDA work group under the auspices of Department of Health and Human Services Task Force on Alzheimer's Disease. Neurology 1984;34:939-44.

23 Kunik ME, Huffman JC, Bharani N, et al. Behavioural disturbances in geropsychiatric inpatients across dementia types. J Geriatr Psychiatry Neurol 2000; 13:49-52.

24 Lyketsos CG, Steinberg M, Tschanz JT, et al. Mental and behavioural disturbances in dementia: findings from the Cache county study on memory and aging. Am J Psychiatry 2000;157:708-14.

25 Cummings JL, Miller B, Hill MA, et al. Neuropsychiatric aspects of multiinfarct dementia and dementia of the Alzheimer type. Arch Neurol 1987;44:389-93

26 Aharon-Peretz J, Masiah A, Pillar T, et al. Sleep-wake cycles in multi-infarct dementia and dementia of the Alzheimer type. Neurology 1991:41:1616-19.

27 Kuzis G, Sabe L, Tiberti C, et al. Neuropsychological correlates of apathy and depression in patients with dementia. Neurology 1999;52:1403-7.

28 Starkstein SE, Fedoroff JP, Price TR, et al. Apathy following cerebrovascular lesions. Stroke 1993;24:1625-31.

29 Cummings JL. Frontal-subcortical circuits and human behavior. Arch Neurol 1993;50:873-80.

30 Foster NL, Chase TN, Fedio P, et al. Alzheimer's disease: focal cortical changes shown by position emission tomography. Neurology 1983;33:961-5.

31 Kertesz A, Clydesdale S. Neuropsychological deficits in vascular dementia vs. Alzheimer's disease. Arch Neurol 1994;51:1226-31.

32 Mega MS, Cummings JL, Fiorello T, et al. The spectrum of behavioral changes in Alzheimer's disease. Neurology 1996:46:130-5.

33 Fuh JL, Liu CK, Mega MS, et al. Behavioral disorders and caregivers reaction in Taiwanese patients with Alzheimer's disease. Int Psychogeriatr 2001;13:121-8. 